\title{
The role of brimonidine tartrate gel in the treatment of rosacea
}

This article was published in the following Dove Press journal:

Clinical, Cosmetic and Investigational Dermatology

23 October 2015

Number of times this article has been viewed

J Mark Jackson'

Melissa Knuckles ${ }^{2}$

John Paul Minni ${ }^{3}$

Sandra Marchese Johnson ${ }^{4}$

Kevin Tate Belasco ${ }^{5}$

'Division of Dermatology, University of Louisville, Louisville, KY, USA; ${ }^{2}$ PSC, Dermatology, Corbin, KY, USA; ${ }^{3}$ Waters Edge Dermatology, Stuart, FL, USA; ${ }^{4}$ Johnson Dermatology, Fort Smith, AR, USA; ${ }^{5}$ Blue Harbor Dermatology, Newport Beach, CA, USA
Correspondence: J Mark Jackson Division of Dermatology, University of Louisville, 50I South 2nd Street, Louisville, KY 40202, USA

Tel +I 5025837546 ext I83

Fax + $502583017 \mid$

Email jacksonjmark@gmail.com
Abstract: Rosacea is a chronic cutaneous condition with a prevalence rate ranging from $9.6 \%$ to $22 \%$ in recent studies. Facial erythema (transient and permanent) is considered a common denominator that is frequently observed in all subtypes of rosacea and is estimated to affect more than 40 million people worldwide. Brimonidine tartrate is a selective $\alpha_{2}$-adrenergic receptor agonist and is the first topical treatment approved for facial erythema of rosacea. Clinical trials have demonstrated that brimonidine tartrate provided significantly greater efficacy, compared to vehicle, for the treatment of moderate to severe erythema of rosacea. In addition, brimonidine tartrate has demonstrated a rapid onset of effect, duration of action throughout the day, and good safety profile in studies of up to 1 year. This review critically discusses the role of brimonidine tartrate for the treatment of facial erythema of rosacea by examining both clinical study data and real-world dermatologist experiences across a wide spectrum of treated patients, and concludes that it is a significant therapeutic option in the management of an unmet need of this chronic condition.

Keywords: rosacea, brimonidine tartrate, erythema, adverse event

\section{Introduction}

Rosacea is a chronic cutaneous condition, characterized by erythema, telangiectasia, papules, pustules, phymatous changes, and ocular involvement. ${ }^{1} \mathrm{~A}$ classification system was devised by the National Rosacea Society (NRS) Expert Committee in 2002, which grouped common signs and symptoms into four subtypes: erythematotelangiectatic (ETR); papulopustular (PPR); phymatous (PHY); and ocular. ${ }^{1}$ ETR is characterized mainly by flushing and persistent facial erythema, whereas PPR is characterized by persistent facial erythema with transient papules and pustules. The presence of thickening skin typifies the PHY subtype, while ocular signs and symptoms in the presence of ETR or PPR are classed as ocular rosacea. ${ }^{1}$ In clinical practice, however, patients often present with a range of symptoms that encompass more than one of these characterizations, ${ }^{1,2}$ although facial erythema (transient and persistent) is a common denominator that is observed often in all subtypes. ${ }^{3}$

The onset of rosacea typically occurs after the age of 30 years, with a peak prevalence around 50 years of age and an increasing prevalence has been noted with advancing age. ${ }^{4-7}$ The sex distribution in rosacea has been variably reported as equal or female predominant. ${ }^{8}$ Rosacea is typically seen in fair-skinned individuals, but has been observed in a variety of skin types and ethnicities. ${ }^{1,5}$

It is estimated that more than 40 million people worldwide are affected by facial erythema of rosacea. ${ }^{9}$ The NRS estimates that 16 million Americans suffer from 
the signs and symptoms of rosacea, and this figure does not include those who are in temporary remission. ${ }^{10}$ The prevalence of rosacea is considered to be increasing in the United States, although this may be attributable to an aging population rather than an increase in incidence. ${ }^{4}$ Various studies have reported the prevalence of rosacea, but many studies have not used an accurate definition of the disease subtypes and/or have used different populations, leading to a wide variation in data within the literature. Recent studies have used the clinical subtypes of rosacea as defined by the NRS, ${ }^{1,11}$ providing what is considered to be more robust data from which we suggest an average prevalence rate of $10 \%$ (with a range from $9.6 \%-22 \%$ ). ${ }^{6,12-15}$

With regards to subtype prevalence, an observational cross-sectional survey of associations between the different subtypes found ETR to be the most prevalent subtype (64\%), with PPR and ocular rosacea both occurring in 36\% of the study population and PHY the least common subtype $(24 \%){ }^{3}$ In this same study, persistent erythema was observed in all subjects and transient erythema was observed at a greater frequency in patients with ETR compared to those with PPR (71\% versus 56\%, respectively; $P=0.08$ ). Papules and pustules were present year-round in $58 \%$ of patients with PPR and were associated with seasonal flares in $74 \%$ of patients with PPR. ${ }^{3}$

In clinical practice, rosacea tends to be underdiagnosed or misdiagnosed. ${ }^{3,16}$ Doctors may not preemptively discuss rosacea with their patients, and studies have shown that only $10 \%$ of patients actively seek professional care. ${ }^{17}$ However, rosacea is known to affect both social and professional lives and can lead to psychosocial problems - surveys have reported that $41 \%$ of rosacea sufferers avoid social and public contact and $76 \%$ experience lowered self-esteem. ${ }^{17}$ In addition, rosacea sufferers also have to contend with commonly associated stigmas: facial erythema incorrectly signifying alcohol abuse or an inability to cope with stress; ${ }^{17}$ and the presence of papules and pustules being mistaken for a lack of personal hygiene. ${ }^{18}$

One of the challenges in treating rosacea lies in the need to alleviate the range of symptoms that can present simultaneously, such as facial erythema and papules and pustules. ${ }^{2}$ An approach involving the use of concomitant therapies for the treatment of different symptoms would help attain the optimum results for the patient with a range of symptoms of rosacea.

Prior to the introduction of brimonidine tartrate, a selective $\alpha_{2}$-adrenergic receptor agonist, there were no approved topical treatments for facial erythema of rosacea. ${ }^{19}$
Avoiding trigger factors, applying sunscreen, camouflage (green tint), or makeup are recommended. ${ }^{2}$ Brimonidine tartrate is the first treatment to offer patients a viable topical prescription to relieve this debilitating symptom. Another $\alpha$-adrenergic receptor agonist, oxymetazoline (an $\alpha_{1}$-adrenergic receptor agonist), is under investigation in Phase III clinical trials for the topical treatment of facial erythema of rosacea. ${ }^{9}$

Brimonidine tartrate was effective and safe, with a rapid onset, in clinical studies up to 1 year for the treatment of facial erythema of rosacea. ${ }^{20-22}$ The most commonly reported adverse event was worsening of erythema and/or flushing, with an approximate incidence of 3\%-7\% in the two 1-month long pivotal studies, and up to $9.1 \%$ in a long-term (1-year) safety study. ${ }^{20-22}$ One year since its approval, several case study reports have confirmed this potential safety observation in real-world practice. ${ }^{23,24}$ However, this effect may be minimized by use of a test area and effective counseling of the patient. ${ }^{24}$

This article will critically review the role of brimonidine tartrate for the treatment of facial erythema of rosacea by first considering the pathophysiology and current treatment strategies of this cutaneous condition, and by secondly examining its action in both clinical studies and real-world dermatologist experiences across a wide spectrum of treated patients.

\section{The pathophysiology of rosacea and current treatment strategies: focus on vasoconstrictive therapy Pathophysiology}

The signs and symptoms of rosacea vary widely between patients and the precise pathophysiological mechanisms in each individual can also vary, depending on the range of clinical manifestations present. ${ }^{25}$ As the most common primary feature of all subtypes of rosacea, facial erythema is recognized as a mandatory diagnostic feature. ${ }^{3,19}$ Facial erythema of rosacea presents as three distinct forms: transient (flushing); nontransient (persistent); and perilesional. The latter occurs as focal zones of erythema surrounding individual papules and pustules and can contribute to the overall appearance of facial redness. ${ }^{19,26}$ Both flushing and persistent facial erythema are distinct from perilesional erythema in that they predominantly occur in a central facial position, are usually diffuse in nature, and tend to persist despite resolution of inflammatory lesions. ${ }^{19,26}$

Although the pathophysiology is yet to be fully understood, the key elements that are often present are inflammation 
and vascular abnormalities. ${ }^{19,25}$ This is confirmed by research showing an upregulation of genes involved in neurogenic inflammation and vasoregulation. ${ }^{27}$ Another of the key changes in patients with rosacea is an increase in vasodilatory activity in response to various trigger factors, such as heat, ultraviolet exposure, exercise, alcohol, and spicy food. ${ }^{26}$ Various microbes, such as Demodex folliculorum, have also been implicated in the pathophysiology of rosacea. ${ }^{28,29}$ An important role has not been identified for known microbial agents in the early phases of rosacea, but they are believed to trigger the innate immunological response, which is both dysregulated and amplified in rosacea, rather than be a mandatory component of disease pathogenesis. ${ }^{28,29}$ In addition, Bacillus oleronius has been isolated from a Demodex mite from a patient with PPR and the potential to stimulate an inflammatory response in patients with PPR has been proposed. ${ }^{30}$ However, further research is needed to verify whether bacteria such as $B$. oleronius play a role in the induction and persistence of rosacea. ${ }^{31}$

A number of different factors are involved in the dysregulation of the inflammatory response in rosacea including cytokines, chemokines, metalloproteinases, proteases, reactive oxygen species molecules, and lipid mediators. A predominantly perivascular inflammatory infiltrate of lymphocytes, macrophages and mast cells has been observed in patients with ETR. ${ }^{25,26}$ In PPR patients, a mixed inflammatory infiltrate of neutrophils, mast cells and, to a lesser degree, eosinophils have been recorded. ${ }^{25}$ It is thought that innate and adaptive immune responses are at play in the PPR subtype through the observation of overexpression of cathelicidin, serine protease, TLR-2, and interleukin- $8 .{ }^{26}$ However, a systematic profile of the inflammatory mediators involved in rosacea pathophysiology at both the gene and protein level is still required. ${ }^{29}$

Characteristic vascular changes in patients with ETR include enlarged, dilated vessels in the upper dermis, ${ }^{25}$ resulting from either neuronal stimulation or inflammatory mediators. ${ }^{29}$ In addition to vascular enlargement, hyperpermeability, and fluid extravasation, markers for angiogenesis and lymphangiogenesis have been observed to increase in rosacea. ${ }^{26}$ Trigger factors, such as heat and certain foods, activate transient receptor vanilloid (TRPV) ion channels, which are overexpressed in patients with the ETR, PPR, and PHY subtypes. ${ }^{32}$ TRPV1 is involved in vasoregulation and is overexpressed on the sensory nerve endings of rosacea patients. It is therefore suggested that neurovascular dysregulation through TRPV1 may also contribute to the sustained periods of transient flushing observed in rosacea patients. ${ }^{29}$
Molecular studies have also identified the significant enhancement of vasoactive neuropeptides, such as adrenergic receptors, in rosacea patients. ${ }^{27}$ The resulting chronic vasodilation and angiogenesis ultimately progress to fixed changes in the vasculature, such as larger, dilated cutaneous vessels, and telangiectasias, leading to persistent erythema. ${ }^{26}$

\section{Current treatment strategies}

Rosacea is considered to be a treatable rather than curable condition, and the identification and avoidance of trigger factors may improve signs and symptoms in some patients. ${ }^{33}$ It is recommended that treatment should be tailored to each individual patient, taking into account the precise symptoms, trigger factors, and psychological and psychosocial impacts of the disease. ${ }^{33}$ In addition, a combination of clinical therapies to treat different symptoms concomitantly may need to be considered to provide the best possible outcomes for the patient.

Current treatment strategies that have received US Food and Drug Administration (FDA) approval for the treatment of rosacea are:

- metronidazole $(0.75 \%$ gel, cream and lotion twice daily; $1 \%$ gel and cream once daily for patients with PPR) ${ }^{34}$

- azelaic acid (15\% gel twice daily for patients with PPR); ${ }^{35}$

- brimonidine tartrate (Mirvaso ${ }^{\circledR}$; Galderma Laboratories, L.P., Fort Worth, TX, USA; $0.33 \%$ gel once daily for patients with facial erythema of rosacea). ${ }^{36}$ Due to a salt policy enacted in the US and European Union, the strength is expressed in terms of the active moiety (brimonidine $0.33 \%$ ) rather than the salt-strength equivalent that was used in the clinical trials (brimonidine tartrate $0.5 \%$ ). It is, however, the same drug concentration; ${ }^{37}$

- sodium sulfacetamide/sulfur (various formulations for patients with acne rosacea) ${ }^{38}$

- subantimicrobial dose doxycycline (40 mg modifiedrelease capsule once daily for patients with PPR); ${ }^{39}$ and - ivermectin (Soolantra ${ }^{\circledR}$; Galderma Laboratories, L.P.; $1 \%$ cream once daily for the treatment of inflammatory lesions of rosacea). ${ }^{40}$

It should be noted that the list does not include medications approved for the treatment of acne rosacea with ophthalmic presentation. Oxymetazoline, another treatment targeting the adrenergic receptors, is currently under investigation. ${ }^{41}$ Off-label drug therapies for rosacea include retinoids ${ }^{42,43}$ and light/laser therapy. ${ }^{2}$

Most available drugs are not efficacious for persistent erythema. ${ }^{34,35,38,39}$ Data relating to approved agents and off-label medical therapies primarily demonstrate benefits 
for the reduction of inflammatory lesions and associated perilesional erythema. ${ }^{29}$ Although facial erythema is one of the primary features of rosacea, brimonidine tartrate is the only topical prescription medication currently approved specifically for this aspect of the disease.

\section{Brimonidine tartrate: pharmacology, mode of action, and pharmacokinetics}

Brimonidine tartrate is a highly selective $\alpha_{2}$-adrenergic receptor agonist and is 1,000-fold more selective for the $\alpha_{2}$-adrenergic receptor than the $\alpha_{1}$-adrenergic receptor. ${ }^{44}$

Direct vasoconstriction of both the small arteries and veins has been established. Piwnica et $\mathrm{al}^{45}$ demonstrated that brimonidine tartrate is a potent vasoconstrictor of human subcutaneous vessels with a diameter of less than $200 \mu \mathrm{m}$ using an ex vivo wire myography model. Additionally, Piwnica et $\mathrm{al}^{45}$ demonstrated that brimonidine tartrate displays anti-inflammatory properties by the inhibition of edema (when compared to vehicle) in in vivo mouse ear inflammation models.

With regards to absorption, no drug accumulation was observed in plasma in a clinical study in which adult subjects with facial erythema of rosacea received repeated cutaneous applications of brimonidine tartrate $0.5 \%{ }^{44}$ Brimonidine tartrate is metabolized extensively by the liver, and the major route of elimination of brimonidine tartrate and its metabolites is urinary excretion. ${ }^{44}$ Brimonidine tartrate contains the following excipients: carbomer; methylparahydroxybenzoate; phenoxyethanol; glycerol; titanium dioxide; propylene glycol; sodium hydroxide; and purified water. ${ }^{44}$

\section{Comparative efficacy, safety, and tolerability}

Brimonidine tartrate has been evaluated in patients with moderate to severe erythema of rosacea in several Phase II and III clinical trials (Table 1). The severity of erythema was measured using the clinician's erythema assessment (CEA) and patient's self-assessment (PSA) scales (Table 2). ${ }^{21,46}$ Unlike treatments for inflammatory lesions, the primary endpoint for these studies included treatment success based on both clinician and patient assessments.

\section{Two Phase Il studies of brimonidine tartrate gel: pharmacodynamics, safety, and finding the optimal daily dose}

The pharmacodynamics and safety of three concentrations $(0.5 \%, 0.18 \%, 0.07 \%)$ of topical brimonidine tartrate gel were evaluated in a randomized, double-blind, parallel-group, vehicle-controlled study ( study A; $n=122$ ). ${ }^{21}$ Brimonidine tartrate was effective in reducing erythema for the 12 hours of the study in a dose-dependent fashion, and the greatest effect was seen with brimonidine tartrate $0.5 \% .{ }^{21}$ All three concentrations were safe and well tolerated. ${ }^{21}$

In the second Phase II study (study B; $n=269$ ), similar to the results seen with study A, brimonidine tartrate $0.5 \%$ once daily demonstrated a rapid onset, a sustained duration of effect, and a dose-dependent relationship. ${ }^{21}$ All dose regimens were safe and well tolerated with a similar incidence of adverse events among the study drug and vehicle groups. ${ }^{21}$ Brimonidine tartrate $0.5 \%$ gel once daily was chosen as the dose for Phase III evaluation, as this was significantly more efficacious than vehicle gel throughout the study.

\section{Two Phase III studies of brimonidine tartrate gel $0.5 \%$ once daily}

The efficacy and safety of brimonidine tartrate $0.5 \%$ once daily was evaluated in two multicenter, randomized, doubleblind, parallel-group, vehicle-controlled studies. ${ }^{20}$ Adult patients (study A, n=260; study B, $n=293$ ) with moderate to severe erythema of rosacea were randomized to receive brimonidine tartrate $0.5 \%$ or vehicle gel once daily for 4 weeks, followed by a 4 -week follow-up period. ${ }^{20}$ The primary efficacy endpoint was the profile of success (defined as a twograde improvement in both CEA and PSA over 12 hours)

Table I Overview of Phase II and III clinical studies with BT in patients with moderate to severe erythema of rosacea

\begin{tabular}{|c|c|c|c|c|c|}
\hline Study name/Phase & Reference & $\begin{array}{l}\text { Sex (malel } \\
\text { female) }\end{array}$ & $\begin{array}{l}\text { Patients } \\
\text { enrolled (n) }\end{array}$ & Treatment & Study centers \\
\hline Phase II study A & Fowler et $\mathrm{al}^{21}$ & $30 / 92$ & 122 & $\begin{array}{l}\text { One application of BT } 0.5 \% \text {, } \\
0.18 \%, 0.07 \% \text {, or vehicle }\end{array}$ & Five in the United States \\
\hline Phase II study B & Fowler et $\mathrm{al}^{21}$ & $52 / 217$ & 269 & $\begin{array}{l}\text { BT } 0.5 \% \text { od } / \mathrm{bd}, \mathrm{BT} 0.18 \% \text { od } / \mathrm{bd} \text {, } \\
\text { or vehicle od/bd }\end{array}$ & 17 in the United States \\
\hline Phase III study A & Fowler et $\mathrm{al}^{20}$ & $54 / 206$ & 260 & BT $0.5 \%$ od, vehicle od & I5 in the United States and Canada \\
\hline Phase III study B & Fowler et $\mathrm{al}^{20}$ & $80 / 213$ & 293 & BT $0.5 \%$ od, vehicle od & I5 in the United States and Canada \\
\hline I-year study & Moore et $\mathrm{al}^{22}$ & $113 / 336$ & 449 & BT $0.5 \%$ od & 27 in the United States \\
\hline
\end{tabular}

Abbreviations: BT, brimonidine tartrate; $n$, number; od, once daily; bd, twice daily. 
Table 2 CEA and PSA

\begin{tabular}{|c|c|c|}
\hline Scores $^{21,46}$ & CEA $^{21}$ & PSA $^{46}$ \\
\hline $0=$ clear & $\begin{array}{l}\text { Clear skin with no signs of } \\
\text { erythema }\end{array}$ & No redness \\
\hline $\mathrm{I}=$ almost clear & Almost clear; slight redness & Very mild redness \\
\hline $2=$ mild & Mild erythema; definite redness & Mild redness \\
\hline $3=$ moderate & $\begin{array}{l}\text { Moderate erythema; marked } \\
\text { redness }\end{array}$ & Moderate redness \\
\hline $4=$ severe & Severe erythema; fiery redness & Severe redness \\
\hline
\end{tabular}

Notes: Adapted with permission, from: Fowler J, Jarratt M, Moore A, et al Brimonidine Phase II Study Group. Once-daily topical brimonidine tartrate gel $0.5 \%$ is a novel treatment for moderate to severe facial erythema of rosacea: results of two multicentre, randomized and vehicle-controlled studies. British Journal of Dermatology. 2012;166(3):633-64I. (C) 201I The Authors. BJD (c) 201I British Association of Dermatologists. ${ }^{21}$ And adapted with permission, from: Jackson JM, Fowler J, Moore A, et al; Brimonidine Phase III Study Group. Improvement in facial erythema within 30 minutes of initial application of brimonidine tartrate in patients with rosacea.J Drugs Dermatol. 2014;13(6):699-704. Copyright (c) 2014 Journal of Drugs in Dermatology. ${ }^{46}$ Abbreviations: CEA, clinician's erythema assessment; PSA, patient's self-assessment.

on days 1,15 , and 29 (Figure 1A). ${ }^{20}$ A one-grade improvement was considered to be a clinically meaningful result. ${ }^{20}$ On day 29 , the responder rate in study A was significantly greater with brimonidine tartrate gel $0.5 \%$ compared with vehicle gel $(P<0.001)$ : $70.9 \%, 69.3 \%, 63.8 \%$, and $56.7 \%$ of patients had a 1-grade improvement on both CEA and PSA at 3,6, 9, and 12 hours, respectively (versus $32.8 \%, 32.0 \%$, $29.7 \%$, and $30.5 \%$ for vehicle gel). ${ }^{20}$ Similarly, in study B, a significant difference was seen between the two groups, with a responder rate of $71.1 \%, 64.8 \%, 66.9 \%$, and $53.5 \%$ in the brimonidine tartrate gel $0.5 \%$ group at $3,6,9$, and 12 hours, respectively (versus $40.1 \%, 43.0 \%, 39.4 \%$, and $40.1 \%$ for vehicle gel; $P<0.001) .{ }^{20}$ The secondary efficacy endpoint was the 30-minute effect, defined as a one-grade improvement from baseline in both CEA and PSA at 30 minutes on day $1 .^{20}$ Brimonidine tartrate was shown to provide significantly greater efficacy than vehicle at 30 minutes for days 1,15 , and 29 (Figure 1). ${ }^{47}$ In both studies, brimonidine tartrate $0.5 \%$ once daily showed significantly greater efficacy compared with vehicle for all efficacy endpoints, with a faster onset of action and a good safety and tolerability profile. ${ }^{20}$

Additional analyses have shown that the patient-reported measure of satisfaction, patient's assessment of appearance (PAA), was correlated to both subject (PSA) and clinician (CEA) measures of facial erythema. ${ }^{47} \mathrm{~A}$ significantly higher number of patients achieved a one-grade improvement within 30 minutes of application of brimonidine tartrate than vehicle. ${ }^{46}$

\section{Long-term safety and efficacy of brimonidine tartrate gel $0.5 \%$ once daily}

The long-term safety and efficacy of brimonidine tartrate $0.5 \%$ once daily was evaluated in a 12-month, open-label,

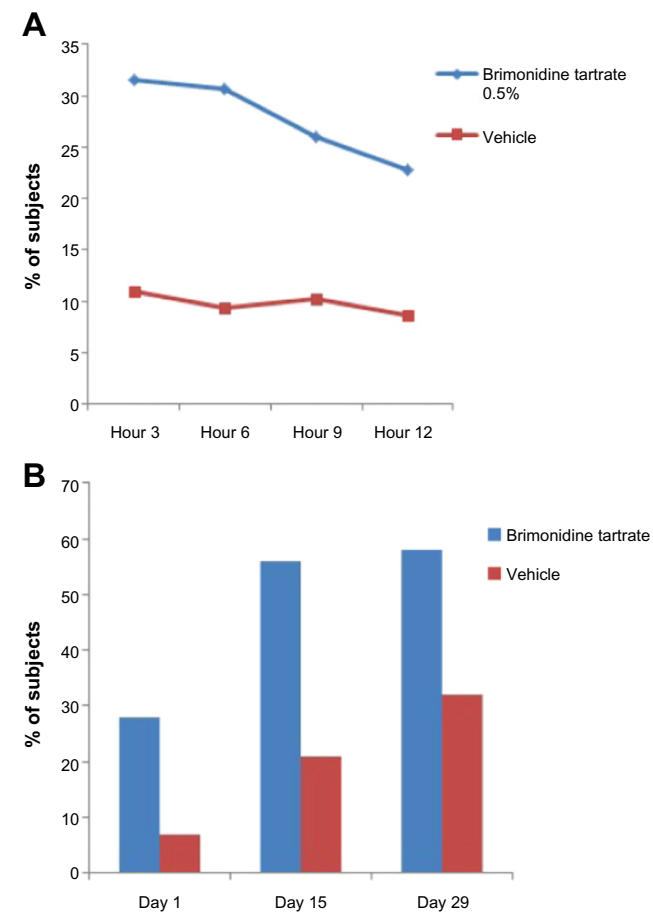

Figure I Percentage of subjects treated with brimonidine tartrate with improvements on CEA and PSA.

Notes: (A) Percentage of subjects having a two-grade improvement on both CEA and PSA on day 29 (treatment with brimonidine tartrate $0.5 \%$ versus vehicle gel); $P<0.00$ I versus vehicle over 12 hours. ${ }^{20}$ Adapted with permission, from: Fowler J Jr, Jackson M, Moore A, et al. Efficacy and safety of once-daily topical brimonidine tartrate gel $0.5 \%$ for the treatment of moderate to severe facial erythema of rosacea: results of two randomized, double-blind, and vehicle-controlled pivotal studies. J Drugs Dermatol. 2013;12(6):650-656. Copyright (C) 2013 Journal of Drugs in Dermatology. ${ }^{20}$ (B) Percentage of subjects having a one-grade improvement on both CEA and PSA in 30 minutes on day I (treatment with brimonidine tartrate $0.5 \%$ versus vehicle gel); $P<0.00$ I versus vehicle at all time points. Adapted with permission, from: Jackson JM, Fowler J, Moore A, et al; Brimonidine Phase III Study Group. Improvement in facial erythema within 30 minutes of initial application of brimonidine tartrate in patients with rosacea. J Drugs Dermatol. 2014;13(6):699-704. ${ }^{46}$ Copyright (C 2014 Journal of Drugs in Dermatology.

Abbreviations: $n$, number; CEA, clinician's erythema assessment; PSA, patient's self-assessment.

multicenter study. ${ }^{22}$ Adult patients $(n=449)$ with moderate to severe erythema of rosacea received brimonidine tartrate $0.5 \%$ once daily for up to 12 months. ${ }^{22}$ Consistent with the results from the previous Phase III trials, effects were seen on day 1 following the first application of brimonidine tartrate (CEA decreased from 3.1 at baseline to 1.7 at hour 3). The improvement in erythema severity was consistently observed throughout the study. ${ }^{22}$ Standardized photographic images of representative subjects prior to and after the application of brimonidine tartrate $0.5 \%$ gel are shown in Figures 2 and 3.

The incidence of adverse events related to the study drug was highest during the first quarter of the study, with most events occurring in the 1 st month. During the second quarter, the adverse events decreased substantially and declined further for the remaining duration of the study. ${ }^{22}$ The majority 


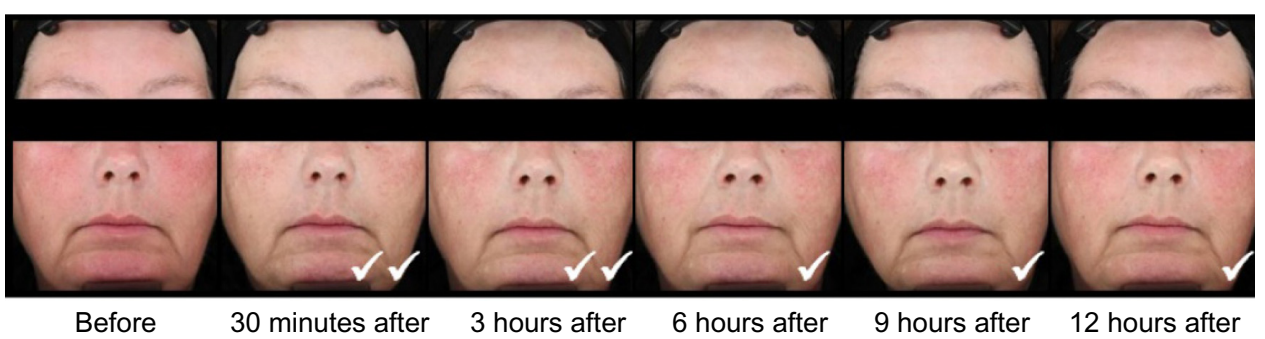

Figure 2 Standardized photos of a representative subject before and at 30 minutes, 3 hours, 6 hours, 9 hours, and 12 hours after the application of brimonidine tartrate gel on day 1 .

Notes: $\checkmark$ One-grade improvement on both CEA and PSA; $\checkmark \checkmark$ two-grade improvement on both CEA and PSA.

Abbreviations: CEA, clinician's erythema assessment; PSA, patient's self-assessment.

of adverse events were dermatologic in nature and mild to moderate in intensity (Table 3), (Galderma Ltd., study number RD.03.DEU.0485.R00, data on file, 2012). ${ }^{3}$ This was the first study to include patients with more than two facial inflammatory lesions and to allow these patients to receive concomitant therapies for their facial papules and pustules. The most common concomitant therapies for papules and pustules are metronidazole, azelaic acid, and tetracycline (ie, tetracycline, minocycline, and doxycycline). ${ }^{21}$ Patients who took other medications during the study did not appear to be at an increased risk of adverse events. ${ }^{22}$

\section{Patient satisfaction}

Rosacea has a high impact on quality of life, ${ }^{27}$ and facial erythema of rosacea in particular can have an impact on selfesteem, in addition to causing embarrassment, anxiety, and avoidance of social situations. These negative emotions can be exacerbated by a range of inaccurate yet commonly associated stigmas from nonsufferers, such as alcohol abuse, stress, anxiousness, and a lack of hygiene, and can lead to reclusive behavior. ${ }^{5,17}$ Despite this observed impact on patients, quality of life is not a commonly evaluated endpoint in rosacea. Of the 58 studies included in the Cochrane review published in 2011, only two studies assessed quality of life as an outcome, and only half of these studies took into account patient assessments of improvements in rosacea severity. ${ }^{48}$ The Cochrane reviewers recommended that future trials should be based on a standardized scale of the patient's assessment of treatment efficacy, with a greater emphasis on changes in quality of life. ${ }^{48}$

The evaluation of brimonidine tartrate in Phase II and III studies included patient-reported assessments (PSA), demonstrating a fast onset of action and long-acting duration..$^{20,21,46}$ In a pooled analysis from the two Phase III studies, ${ }^{20}$ patients were asked to grade their satisfaction of the overall appearance of their skin using the PAA scale. ${ }^{47}$ The PAA can be considered a psychological anchor with five categories ranging from 0 (very satisfied) to 4 (very dissatisfied). ${ }^{47}$ This anchor-based method allows the results to be compared with changes in PSA and CEA scores. ${ }^{47}$ Improvements in PSA and CEA were highly correlated with improvements in PAA. For every time point evaluated, patients who achieved a one- or two-grade improvement in both the CEA and PSA scores were significantly more likely to be satisfied with the appearance of their skin (as measured by the PAA) compared with those subjects who did not achieve an improvement in CEA and PSA $(P<0.001) .{ }^{47}$ This study demonstrated convergent validity of facial erythema, as assessed by the subjects (PSA) and clinicians (CEA), and that these measures correlated with a patient-reported measure of satisfaction (PAA). ${ }^{47}$

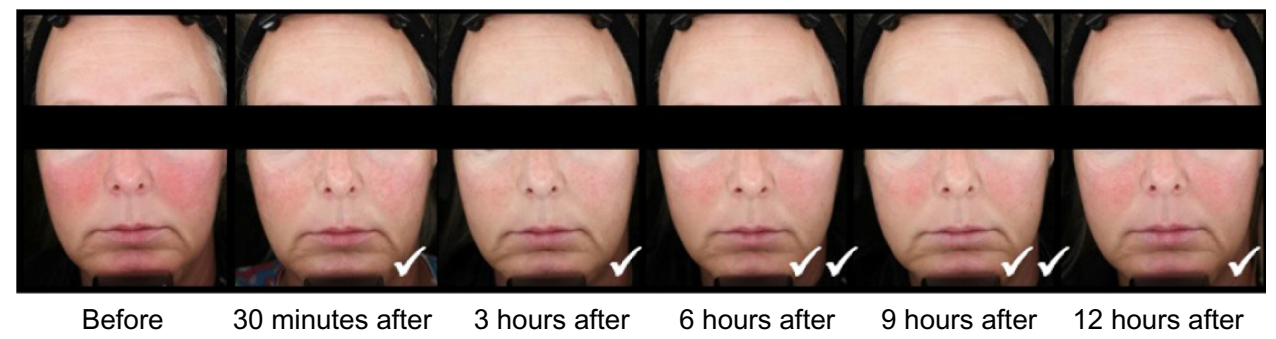

Figure 3 Standardized photos of a representative subject before and at 30 minutes, 3 hours, 6 hours, 9 hours, and 12 hours after the application of brimonidine tartrate gel on day I.

Notes: $\checkmark$ One-grade improvement on both CEA and PSA; $\checkmark \checkmark$ two-grade improvement on both CEA and PSA. C 2014 European Academy of Dermatology and Venereology. Publisher: John Wiley and Sons. Reproduced from Fowler J, Tan J, Jackson JM, et al; Brimonidine Phase III Study Group. Treatment of facial erythema in patients with rosacea with topical brimonidine tartrate: correlation of patient satisfaction with standard clinical endpoints of improvement of facial erythema. Journal of the European Academy of Dermatology and Venereology. 2015;29(3):474-481.47

Abbreviations: CEA, clinician's erythema assessment; PSA, patient's self-assessment. 
Table 3 Most commonly reported adverse events considered to be related to the study drug ( $\geq 2 \%$ patients)

\begin{tabular}{ll}
\hline Adverse event & Percentage of patients \\
\hline Flushing & 9.1 \\
Worsening of erythema & 6.5 \\
Worsening of rosacea & 3.6 \\
Skin burning sensation & 3.3 \\
Skin irritation & 3.1 \\
Contact dermatitis & 2.2 \\
Pruritus & 2.0 \\
\hline
\end{tabular}

Notes: The total number of patients reporting adverse events was 139 (31\%). Data from Galderma Ltd., study number RD.03.DEU.0485.R00, data on file, 2012; and Tan et al. ${ }^{3}$

In the long-term study, patients were asked to rate the impact of rosacea on their social life at various time points. ${ }^{22}$ At baseline, $29.5 \%$ of patients considered that their rosacea inhibited their social life. This decreased to $14.2 \%$ by month 3 and stabilized throughout the study. ${ }^{22}$ Similar results were seen for the other two elements of the questionnaire: $32.7 \%$ of patients thought that rosacea had caused them to avoid public contact or social engagement at baseline, and this decreased to $13.1 \%-19.4 \%$ for months $3-12$ of the study. In addition, $17.3 \%$ of patients considered rosacea as the cause of difficulty in establishing new relationships at baseline, decreasing to $7.1 \%-11.1 \%$ after $3-12$ months of treatment with brimonidine tartrate. ${ }^{22}$

Patient-reported outcomes have been investigated in adult subjects $(n=92)$ with moderate-severe facial erythema of rosacea following treatment with brimonidine tartrate $0.33 \%$ versus vehicle. A multicenter, randomized, placebocontrolled, double-blind, and parallel-group study showed that subjects treated with brimonidine tartrate $0.33 \%$ were significantly more satisfied (all $P<0.01$ ) with the overall treatment (69.6\% versus $40.4 \%)$, improvement of facial erythema (67.4\% versus $33.3 \%$ ), and time to treatment effect $(69.5 \%$ versus $33.3 \%)$ compared to vehicle, respectively. Significantly more subjects in the study drug group thought that their facial appearance improved since starting the treatment (63.0\% versus $26.8 \%$, respectively; $P<0.05$ ), and they agreed that they were able to control their facial erythema $(60.0 \%$ versus $33.3 \%$, respectively; $P<0.05){ }^{49}$

\section{Real-world experiences of brimonidine tartrate for the treatment of facial erythema of rosacea}

The results detailed from the clinical studies are those seen in the authors' clinical practice as well, with more than $85 \%$ of patients displaying positive responses to brimonidine tartrate.
In our clinics, we inform patients that brimonidine tartrate is a vasoactive topical medication, and that the results can be variable. It is important to stress that some patients may not respond as quickly as others, and that a level of patience may be required. As a symptomatic therapy, patients also need to understand that facial erythema will return once the effects of the drug have worn off. Applying brimonidine tartrate will not completely negate the erythema-inducing effects of rosacea triggers, such as spicy foods and alcohol, and so it needs to be made clear to the patient to identify and avoid their potential rosacea triggers.

Emphasizing that this clinical therapy is not intended to eradicate papules, pustules or phymatous changes of rosacea, or to eradicate oiliness on the face, is important. We find that combination therapy of brimonidine tartrate with treatments, for example, for inflammatory lesions, can be beneficial in addressing multiple symptoms of rosacea.

Time needs to be taken to discuss the application of the drug. We recommend that patients apply a very small, peasized amount of the drug at first and spread it uniformly over the face once daily. Thorough and uniform spreading of the product can prevent a streaky or patchy appearance, and the use of other daily routine products, such as sunscreen and cosmetics, is recommended for application after the brimonidine tartrate has dried off.

In our clinics, approximately $4 \%-15 \%$ of patients have complained of worsening erythema or flushing, and some of these patients decided to discontinue treatment with brimonidine tartrate, which led to resolution of these side effects usually within 4-24 hours. Many patients, however, have noted that the benefits outweigh these side effects and they elected to continue treatment, as the worsening of erythema was only temporary and not a daily issue. In addition, these adverse events often occurred during the 1 st week and decreased with continued use. Sometimes the initial cause of the flare was that the patient had applied too much of the medication, too frequently, without applying sunscreen or without continuing to avoid common triggers of rosacea flushing. This highlights the importance of providing clear and comprehensive counseling to the patient.

\section{Summary and conclusion}

Brimonidine tartrate offers clinicians the first topically effective agent for the treatment of facial erythema of rosacea, providing a rapid-onset effect, sustained duration, and good tolerability from both clinical trials and real-world experiences.

Rosacea is a common condition ${ }^{1,10}$ that is often underdiagnosed by doctors. ${ }^{16}$ Patients not only have the burden of physical manifestations to cope with, but also the psychosocial 
impact of the condition. The erythema of rosacea, in particular, has been associated with a detrimental effect on quality of life, including low self-esteem, anxiety, and avoidance of social situations. ${ }^{17}$

The pathophysiology of the disease remains to be fully elucidated, and can vary depending on the precise clinical features in each individual. ${ }^{25}$ The key pathophysiological elements are inflammation and vascular abnormalities. ${ }^{19,25}$ Treatment strategies for rosacea should be tailored to each individual patient, depending on the precise symptoms presented. ${ }^{33}$ The selective $\alpha_{2}$-adrenergic receptor agonist, brimonidine tartrate, is the first US FDA-approved therapy for the treatment of facial erythema and it provides a clinical option to alleviate this common, primary feature of rosacea. Brimonidine tartrate has been shown to be effective and safe, with a rapid onset, in Phase II and III studies for the treatment of facial erythema of rosacea. ${ }^{20-22,46}$

The stringency of the evaluation in the studies underestimates the true clinical effect of brimonidine tartrate, as patients and clinicians were not able to rely on baseline photos when performing evaluations. This resulted in difficulty obtaining a two-grade improvement, as many patients were not able to note the changes in their appearance without seeing the baseline photos. As the study progressed and patients were able to see the medication wear off, and they then reapplied the medication to see the effects the next day, they were better able to note the improvement. It should also be noted that the scales used for the evaluation of erythema have very subtle differences between them, making it even more difficult to demonstrate a two-grade improvement. For example, a patient with a CEA and PSA of 3 at baseline who still had some minimal erythema may have been graded as a 2 based on the scale, but based on the subtle differences, this same patient could have also been graded as a 1 on one or both scales. Therefore, the true measure of response is having our patients see the effects of the medication and its duration of response, and more than $85 \%$ of patients in our clinical practices have displayed a positive response to brimonidine tartrate.

In the long-term safety study, the majority of the adverse events occurred during the 1 st month. The majority of subjects with adverse events remained in the study, which may signify that the patients appreciated the benefit of the treatment despite the adverse event that they experienced. ${ }^{22}$ The long-term evaluation also demonstrated that brimonidine tartrate is safe and well tolerated, even in the presence of concomitant medications specific for other rosacea symptoms (such as papules and pustules). ${ }^{22}$ This finding suggests that brimonidine tartrate provides a symptomatic treatment for the underlying nontransient erythema in all rosacea patients. It may be a single treatment or combined with an anti-inflammatory treatment for PPR. These approaches are used in the authors' clinical practice.

There is no cure for the facial erythema of rosacea, and until now, very little has demonstrated improvement in this manifestation. Some of the currently approved agents demonstrate an effect on perilesional erythema, but brimonidine tartrate is the first to demonstrate improvement in the persistent erythema of rosacea. Once the effects of brimonidine tartrate treatment wear off, the symptoms reoccur.

Brimonidine tartrate has been associated with temporary worsening of erythema in a few cases (approximately 12 hours after the initial application). ${ }^{23,24}$ However, this is only a small proportion of patients who have been treated with brimonidine tartrate since its launch over 1 year ago. It has been suggested that an atypical pattern of vascular reactivity and response, or flare of facial vasodilation (flushing) induced by an exogenous trigger factors, are some of the most likely causes for such cases. ${ }^{50}$

In light of this, the importance of counseling patients and setting appropriate patient expectations prior to treatment initiation cannot be underestimated. ${ }^{23,24}$ One case study reported allergic contact dermatitis in a patient several days after treatment with brimonidine tartrate. ${ }^{51} \mathrm{~A}$ small proportion of patients developing contact dermatitis associated with brimonidine tartrate treatment is not surprising, when it is considered that contact dermatitis was reported in $2.2 \%$ of subjects treated with brimonidine tartrate in the long-term (52-week) study of subjects with facial erythema of rosacea. ${ }^{22,50}$ In addition, approximately $1 \%$ of subjects were reported to develop allergic contact dermatitis based on data completed in clinical trials during the drug development process. ${ }^{22,50}$

In order to see the true measure of response on an individual basis, a small sample of brimonidine tartrate can be provided for the patient to try at home and to monitor their response over a few days. In the authors' opinion, there has been a predictable response in each individual patient, and this response has continued with subsequent applications. Most of the adverse events related to brimonidine tartrate have occurred within the first 1-2 weeks, and none of the adverse events have produced long-term sequelae that adversely affect the course of the disease. One might argue that regular use of brimonidine tartrate gel for ETR might alter the course of the disease over time. This is a hypothetical question that may be answered with future studies.

Given the debilitating impact of facial erythema on quality of life, an effective treatment that provides a rapid and sustained effect over 12 hours, with a good tolerability 
and safety profile, is central to the needs of rosacea patients. Brimonidine tartrate is a significant drug in the management of erythema of rosacea which, at this point, has not had any effective therapy.

\section{Acknowledgments}

Medical writing support was provided by Natasha Singh Kent, PhD; Gavin Kenny, PhD; and Jen Lewis, PhD, at Havas Life Medicom and was funded by Galderma. The studies were sponsored by Galderma R\&D.

\section{Disclosure}

Doctors Jackson, Johnson, and Belasco have served as investigators and advisors for Galderma and participated in meetings as speakers for Galderma. Doctor Minni has participated as a speaker for Galderma, AbbVie, Allergan, Johnson and Johnson, Promius, and Valeant. The authors report no other conflicts of interest in this work.

\section{References}

1. Wilkin J, Dahl M, Detmar M, et al. Standard classification of rosacea: Report of the National Rosacea Society Expert Committee on the Classification and Staging of Rosacea. J Am Acad Dermatol. 2002;46(4): 584-587.

2. Elewski BE, Draelos Z, Dréno B, Jansen T, LaytonA, Picardo M. Rosaceaglobal diversity and optimized outcome: proposed international consensus from the Rosacea International Expert Group. J Eur Acad Dermatol Venereol. 2011;25(2):188-200.

3. Tan J, Blume-Peytavi U, Ortonne JP, et al. An observational crosssectional survey of rosacea: clinical associations and progression between subtypes. Br J Dermatol. 2013;169(3):555-562.

4. Drake L, editor [webpage on the Internet]. Patients over 50 hardest hit with rosacea symptoms. Rosacea Review. Barrington, IL: National Rosacea Society; 2000. Available from: http://www.rosacea.org/rr/2000/ fall/article_2.php. Accessed April 16, 2014.

5. Blount BW, Pelletier AL. Rosacea: a common, yet commonly overlooked, condition. Am Fam Physician. 2002;66(3):435-440.

6. Chosidow O, Cribier B. Epidemiology of rosacea: updated data. Ann Dermatol Venereol. 2011;138 Suppl 3:S179-S183.

7. Abram K, Silm H, Maaroos HI, Oona M. Risk factors associated with rosacea. J Eur Acad Dermatol Venereol. 2010;24(5):565-571.

8. Tan J, Berg M. Rosacea: current state of epidemiology. J Am Acad Dermatol. 2013;69(6 Suppl 1):S27-S35.

9. Shanler SD, Ondo AL. Successful treatment of the erythema and flushing of rosacea using a topically applied selective a1 adrenergic receptor agonist, oxymetazoline [abstract]. $J$ Am Acad Dermatol. 2008:58(2):AB9 P400.

10. Drake L, editor [webpage on the Internet]. Rosacea now estimated to affect at least 16 million Americans. Rosacea Review. Barrington, IL: National Rosacea Society; 2010. Available from: http://www.rosacea. org/rr/2010/winter/article_1.php. Accessed April 16, 2014.

11. Wilkin J, Dahl M, Detmar M, et al; National Rosacea Society Expert Committee. Standard grading system for rosacea: report of the National Rosacea Society Expert Committee on the classification and staging of rosacea. J Am Acad Dermatol. 2004;50(6):907-912.

12. Drake L, editor [webpage on the Internet]. Research shows higher prevalence. Rosacea Review. Barrington, IL: National Rosacea Society; 2008. Available from: http://www.rosacea.org/rr/2008/fall/article_2. php. Accessed April 16, 2014.
13. Drake L, editor [webpage on the Internet]. New studies show high incidence of rosacea and possible new causes. Rosacea Review. Barrington, IL: National Rosacea Society; 2008. Available from: http:// www.rosacea.org/rr/2007/summer/article_1.php. Accessed April 16, 2014.

14. Abram K, Silm H, Oona M. Prevalence of rosacea in an Estonian working population using a standard classification. Acta Derm Venereol. 2010;90(3):269-273.

15. Berg M, Lidén S. An epidemiological study of rosacea. Acta Derm Venereol. 1989;69(5):419-423.

16. Baldwin HE. Diagnosis and treatment of rosacea: state of the art. J Drugs Dermatol. 2012;11(6):725-730.

17. Baldwin H. Psychosocial implications of rosacea. The Dermatologist. 2012;Supplement:2-4.

18. Drake L, editor [webpage on the Internet]. Rosacea sufferers, take heart you are not alone. Rosacea Review. Barrington, IL: National Rosacea Society; 1997. Available from: http://www.rosacea.org/rr/1997/winter/ article_1.php. Accessed September 9, 2014.

19. Del Rosso JQ. Management of facial erythema of rosacea: what is the role of topical $\alpha$-adrenergic receptor agonist therapy? J Am Acad Dermatol. 2013;69(6 Suppl 1):S44-S56.

20. Fowler J Jr, Jackson M, Moore A, et al. Efficacy and safety of oncedaily topical brimonidine tartrate gel $0.5 \%$ for the treatment of moderate to severe facial erythema of rosacea: results of two randomized, double-blind, and vehicle-controlled pivotal studies. J Drugs Dermatol. 2013;12(6):650-656.

21. Fowler J, Jarratt M, Moore A, et al; Brimonidine Phase II Study Group. Once-daily topical brimonidine tartrate gel $0.5 \%$ is a novel treatment for moderate to severe facial erythema of rosacea: results of two multicentre, randomized and vehicle-controlled studies. $\mathrm{Br} J$ Dermatol. 2012;166(3):633-641.

22. Moore A, Kempers S, Murakawa G, et al. Long-term safety and efficacy of once-daily topical brimonidine tartrate gel $0.5 \%$ for the treatment of moderate to severe facial erythema of rosacea: results of a 1-year open-label study. J Drugs Dermatol. 2014;13(1):56-61.

23. Ilkovitch D, Pomerantz RG. Brimonidine effective but may lead to significant rebound erythema. J Am Acad Dermatol. 2014;70(5): e109-e110.

24. Routt ET, Levitt JO. Rebound erythema and burning sensation from a new topical brimonidine tartrate gel $0.33 \%$. J Am Acad Dermatol. 2014;70(2):e37-e38.

25. Cribier B. Rosacea under the microscope: characteristic histological findings. J Eur Acad Dermatol Venereol. 2013;27(11):1336-1343.

26. Del Rosso JQ. Advances in understanding and managing rosacea: part 1: connecting the dots between pathophysiological mechanisms and common clinical features of rosacea with emphasis on vascular changes and facial erythema. J Clin Aesthet Dermatol. 2012;5(3):16-25.

27. Schwab VD, Sulk M, Seeliger S, et al. Neurovascular and neuroimmune aspects in the pathophysiology of rosacea. J Investig Dermatol Symp Proc. 2011;15(1):53-62.

28. Del Rosso JQ, Gallo RL, Kircik L, Thiboutot D, Baldwin HE, Cohen D. Why is rosacea considered to be an inflammatory disorder? The primary role, clinical relevance, and therapeutic correlations of abnormal innate immune response in rosacea-prone skin. J Drugs Dermatol. 2012;11(6): 694-700.

29. Steinhoff M, Buddenkotte J, Aubert J, et al. Clinical, cellular, and molecular aspects in the pathophysiology of rosacea. J Investig Dermatol Symp Proc. 2011;15(1):2-11.

30. Lacey N, Delaney S, Kavanagh K, Powell FC. Mite-related bacterial antigens stimulate inflammatory cells in rosacea. Br J Dermatol. 2007;157(3):474-481.

31. Jarmuda S, O'Reilly N, Zaba R, Jakubowicz O, Szkaradkiewicz A, Kavanagh K. Potential role of Demodex mites and bacteria in the induction of rosacea. J Med Microbiol. 2012;61(Pt 11):1504-1510.

32. Sulk M, Seeliger S, Aubert J, et al. Distribution and expression of nonneuronal transient receptor potential (TRPV) ion channels in rosacea. J Invest Dermatol. 2012;132(4):1253-1262. 
33. Odom R, Dahl M, Dover J, et al; National Rosacea Society Expert Committee on the Classification and Staging of Roasacea. Standard management options for rosacea, part 1: overview and broad spectrum of care. Cutis. 2009;84(1):43-47.

34. Wolf JE Jr, Del Rosso JQ. The CLEAR trial: results of a large community-based study of metronidazole gel in rosacea. Cutis. 2007;79(1): 73-80.

35. Thiboutot D, Thieroff-Ekerdt R, Graupe K. Efficacy and safety of azelaic acid $(15 \%)$ gel as a new treatment for papulopustular rosacea: results from two vehicle-controlled, randomized phase III studies. J Am Acad Dermatol. 2003;48(6):836-845.

36. European Medicines Agency. Assessment Report (EMEA/ CHMP/115246/2014). London, UK: European Medicines Agency; 2013. Available from: http://www.ema.europa.eu/docs/en_GB/ document_library/EPAR_-_Public_assessment_report/human/002642/ WC500163196.pdf. Accessed April 16, 2014.

37. Center for Drug Evaluation and Research Policies and Procedures. Naming of Drug Product Containing Salt Drug Substances. Silver Spring, MD: United States Food and Drug Administration; 2013. Available from: http://www.fda.gov/downloads/AboutFDA/ CentersOffices/OfficeofMedicalProductsandTobacco/CDER/ ManualofPoliciesProcedures/UCM340273.pdf. Accessed April 16, 2014.

38. Sauder DN, Miller R, Gratton D, Danby W, Griffiths C, Phillips SB. The treatment of rosacea: the safety and efficacy of sodium sulfacetamide $10 \%$ and sulfur 5\% lotion (Novacet) is demonstrated in a double-blind study. J Dermatolog Treat. 1997;8(2):79-85.

39. Del Rosso JQ, Webster GF, Jackson M, et al. Two randomized phase III clinical trials evaluating anti-inflammatory dose doxycycline (40-mg doxycycline, USP capsules) administered once daily for treatment of rosacea. J Am Acad Dermatol. 2007;56(5):791-802.

40. Soolantra ${ }^{\circledR}$ (ivermctin) [prescribing information]. Forth Worth, TX: Galderma Laboratories, L.P.; 2015.

41. Shanler SD, Ondo AL. Successful treatment of the erythema and flushing of rosacea using a topically applied selective alpha1-adrenergic receptor agonist, oxymetazoline. Arch Dermatol. 2007;143(11):1369-1371.
42. Altinyazar HC, Koca R, Tekin NS, Eştürk E. Adapalene vs metronidazole gel for the treatment of rosacea. Int J Dermatol. 2005;44(3):252-255.

43. Gollnick H, Blume-Peytavi U, Szabó EL, et al. Systemic isotretinoin in the treatment of rosacea-doxycycline- and placebo-controlled, randomized clinical study. J Dtsch Dermatol Ges. 2010;8(7):505-515.

44. electronic Medicines Compendium (eMC) [webpage on the Internet]. Mirvaso 3mg/g gel. Uxbridge, UK: electronic Medicines Compendium (eMC); 2014. Available from: http://www.medicines.org.uk/emc/ medicine/28682/SPC/Mirvaso+3mg+g+Gel/\#PHARMACOLOGIC AL_PROPS. Accessed April 18, 2014.

45. Piwnica D, Rosignoli C, de Ménonville ST, et al. Vasoconstriction and anti-inflammatory properties of the selective $\alpha$-adrenergic receptor agonist brimonidine. J Dermatol Sci. 2014;75(1):49-54.

46. Jackson JM, Fowler J, Moore A, et al; Brimonidine Phase III Study Group. Improvement in facial erythema within 30 minutes of initial application of brimonidine tartrate in patients with rosacea. $J$ Drugs Dermatol. 2014;13(6):699-704

47. Fowler J, Tan J, Jackson JM, et al; Brimonidine Phase III Study Group. Treatment of facial erythema in patients with rosacea with topical brimonidine tartrate: correlation of patient satisfaction with standard clinical endpoints of improvement of facial erythema. J Eur Acad Dermatol Venereol. 2015;29(3):474-481.

48. van Zuuren EJ, Kramer S, Carter B, Graber MA, Fedorowicz Z. Interventions for rosacea. Cochrane Database Syst Rev. 2011;(3): CD003262.

49. Layton A, Schaller M, Homey B, et al. Brimonidine $3 \mathrm{mg} / \mathrm{g}$ gel improves patient-reported outcomes in severe facial erythema of rosacea. Poster presented at: 23rd EADV Congress Building Bridges; October 8-12; 2014; Amsterdam, the Netherlands.

50. Del Rosso JQ. Reply to "Allergic contact dermatitis to topical brimonidine tartrate gel $0.33 \%$ for treatment of rosacea". J Am Acad Dermatol. 2014;71(4):833-834.

51. Swanson LA, Warshaw EM. Allergic contact dermatitis to topical brimonidine tartrate gel $0.33 \%$ for treatment of rosacea. $\mathrm{J} \mathrm{Am} \mathrm{Acad}$ Dermatol. 2014;71(4):832-833.
Clinical, Cosmetic and Investigational Dermatology

\section{Publish your work in this journal}

Clinical, Cosmetic and Investigational Dermatology is an international, peer-reviewed, open access, online journal that focuses on the latest clinical and experimental research in all aspects of skin disease and cosmetic interventions. All areas of dermatology will be covered; contributions will be welcomed from all clinicians and

\section{Dovepress}

basic science researchers globally. This journal is indexed on CAS The manuscript management system is completely online and includes a very quick and fair peer-review system, which is all easy to use. Visit http://www.dovepress.com/testimonials.php to read real quotes from published authors. 